\title{
Integrative systems medicine approaches to identify molecular targets in lymphoid malignancies
}

\author{
Raffaele Frazzi ${ }^{1}$, Charles Auffray ${ }^{2}$, Angela Ferrari ${ }^{3}$, Perla Filippini ${ }^{4}$, Sergio Rutella ${ }^{5^{*}}$ (D) and Alfredo Cesario $6,7,8$
}

\begin{abstract}
Although survival rates for lymphoproliferative disorders are steadily increasing both in the US and in Europe, there is need for optimizing front-line therapies and developing more effective salvage strategies. Recent advances in molecular genetics have highlighted the biological diversity of lymphoproliferative disorders. In particular, integrative approaches including whole genome sequencing, whole exome sequencing, and transcriptome or RNA sequencing have been instrumental to the identification of molecular targets for treatment. Herein, we will discuss how genomic, epigenomic and proteomic approaches in lymphoproliferative disorders have supported the discovery of molecular lesions and their therapeutic targeting in the clinic.
\end{abstract}

Keywords: Systems medicine, Lymphoproliferative disorder, Massively-Parallel sequencing, High throughput RNA sequencing

\section{Background}

The growing medical needs in terms of cost reduction, care of elderly patients, increasing quality requirements and management, pose new hurdles to health professionals. Systems medicine (SM) is a fascinating approach to provide answers to these challenges. SM can be defined as an interdisciplinary, pro-active approach to the treatment of diseases that follows the P4 paradigm: predictive, preventive, personalized and participatory $[1,2]$. SM takes advantage of a systems biology approach, whereby biological, clinical, environmental and lifestyle information is integrated through iterative statistical analyses, computational modelling and experimental validation [1].

A variety of hematological malignancies have been characterised, at least partially, using whole genome sequencing (WGS), whole exome sequencing (WES), transcriptome or RNA sequencing, including acute and chronic leukemias and lymphomas. The data derived

\footnotetext{
*Correspondence: sergio.rutella@ntu.ac.uk

5 John van Geest Cancer Research Centre, College of Science and Technology, Nottingham Trent University, Clifton Campus, Nottingham NG11 8NS, UK

Full list of author information is available at the end of the article
}

from these efforts have provided pathogenetic insights, as well as diagnostic and therapeutic information, and they have identified molecular lesions amenable to targeted interventions. Next generation sequencing (NGS) or massively parallel sequencing refers to a technology which does not depend on traditional electrophoresisbased Sanger sequencing (i.e., the first generation) [3]. NGS platforms can be broadly divided into second generation (i.e., methods requiring template amplification before sequencing) and third generation (i.e., methods that sequence templates directly without amplification). Sophisticated computational tools are required to map and assemble the massive amount of data generated from a single NGS reaction.

The management of chronic lymphoproliferative disorders is a considerable burden on public health [4]. Non-Hodgkin lymphomas (NHL) are most frequently diagnosed in patients aged 65-74 (median age at diagnosis being 66 years) and account for $4.3 \%$ of new cancer cases in the US [5]. NHLs are, therefore, the seventh most common type of cancer diagnosed in the US. The population age is increasing in Western countries, implying that the impact of healthcare costs necessary to cure these malignancies will be increasing as well. Death rates 
have been declining by $2.6 \%$ on average each year during 2002-2011. The 5-year survival rate for NHLs currently approaches $70 \%$ [5]. Specifically, survival rates for follicular lymphoma (FL) increased from 59 to $74 \%$, and those for chronic lymphocytic leukemia (CLL)/small lymphocytic lymphoma (SLL) from 66 to 69 \% [4]. Similarly, EUROCARE and HAEMACARE data on 560,444 patients with haematological malignancies show an increased survival for most disease types in European countries, with the largest increase being for diffuse large B-cell lymphoma (DLBCL) and FL. However, persistent differences in survival across Europe point to the need for minimizing variations in the quality of care and availability of new treatments [4].

NHLs comprise over 60 disease subtypes and include both aggressive and indolent lymphoma subgroups. Approximately $95 \%$ of lymphomas in the Western world are of B-cell origin. This overrepresentation largely originates from misrepair of DNA lesions introduced by activation-induced cytidine deaminase (AID), a B cellspecific cytidine deaminase that initiates class switch recombination and somatic hypermutation (SHM) of immunoglobulin (Ig) genes [6]. The three most common subtypes of NHL are represented by DLBCL, FL and CLL/SLL [7]. This article will discuss how genomic, epigenomic and proteomic approaches have supported the discovery of molecular targets in well-established lymphoid malignancies [8]. The main studies covered in this paper have been summarised in Table 1.

\section{Use of high-throughput approaches to identify molecular targets in DLBCL}

DLBCL is an aggressive type of NHL, accounting for approximately $30 \%$ of adult NHLs diagnosed in Western countries [9]. A breakthrough in the treatment of DLBCL was represented by the use of the monoclonal antibody rituximab in addition to standard $\mathrm{CHOP}$ chemotherapy (R-CHOP), which led to shortening of time to disease progression and to dramatic improvements in overall survival (OS) $[10,11]$.

The cell of origin is an important prognosticator in DLBCLs. Gene expression profiling (GEP) has allowed the identification of three molecular subtypes of DLBCLs, i.e., germinal center B-cell-like DLBCL (GCB), activated B-cell-like (ABC) DLBCLs, and primary mediastinal B-cell lymphoma (PMBL) [12]. High-resolution, genome-wide copy number analysis coupled with GEP has indicated that GCB, ABC and PMBL are different diseases that use distinct oncogenic pathways and have a different prognosis [13].

GEP studies contributed further to the molecular and prognostic classification of DLBCLs through the identification of "stromal-1" and "stromal-2" signatures in a training group as well as a validation cohort of patients [14]. Molecular signatures were related to the microenvironment and predicted either a favorable outcome (stromal-1) by reflecting extracellular matrix deposition and macrophage infiltration of the tumor or a less favorable outcome (stromal-2) as a result of tumor blood vessel density. The stromal- 1 and stromal-2 signature genes were more highly expressed in the nonmalignant CD19fraction compared with $\mathrm{CD} 19^{+}$lymphoma cells. These signatures were found both in GCB-DLBCL and in ABCDLBCL, reflecting biological attributes of both subtypes of DLBCL [15].

At genetic level, massive parallel sequencing techniques revealed a remarkable complexity of DLBCLs represented by translocations, gene amplifications, single nucleotide variants but also copy number variations. Interestingly, recent genomic studies unveiled lesions affecting histone/chromatin modification enzymes, such as CREBBP, EP300, MLL and EZH2 [16-18]. A further layer of complexity is represented by methylation profiles analyzed at the genome level. Methylation variability profiles (MVP) and methylation variability scores (MVS) have been recently introduced as novel methods to measure methylation disruption in lymphomas [19]. These parameters describe the deviation of the methylation patterns of DLBCLs from normal GCB cells (NGCB). They have been recently introduced and define six separate clusters in a cohort of 140 DLBCL analyzed. The magnitude of the methylation deviation from NGCB associates with survival of patients receiving R-CHOP, insofar patients with a larger magnitude of methylation changes display poorer survival compared with patients with smaller magnitude of methylation changes [19].

$M Y C$ oncogene rearrangements impart an unfavorable prognosis on lymphomas [20,21]. Furthermore, the prognostic impact of $M Y C$ dysregulation is strongly influenced by concomitant $B C L 2$ aberrations. The concurrent translocation of $M Y C$ and $B C L 2$ occurs in approximately $5 \%$ of DLBCLs and defines "double-hit" lymphomas that are treatment-refractory, with a median survival of $\sim 8$ months $[22,23]$. Dual expression of these proteins is more common in ABC-DLBCLs and may explain its poorer survival [24]. This evidence points to the importance of assessing DLBCL patients for concurrent deregulation of $M Y C$ and $B C L 2$ at time of diagnosis, both at the level of translocations and protein overexpression [15].

Our knowledge of the biological heterogeneity of DLBCLs has prompted the development of novel targeted therapies, that have the potential for greater tumor specificity and lower generalized toxicity. An example is represented by agents that target B-cell receptor (BCR) signaling in DLBCLs. For instance, members 


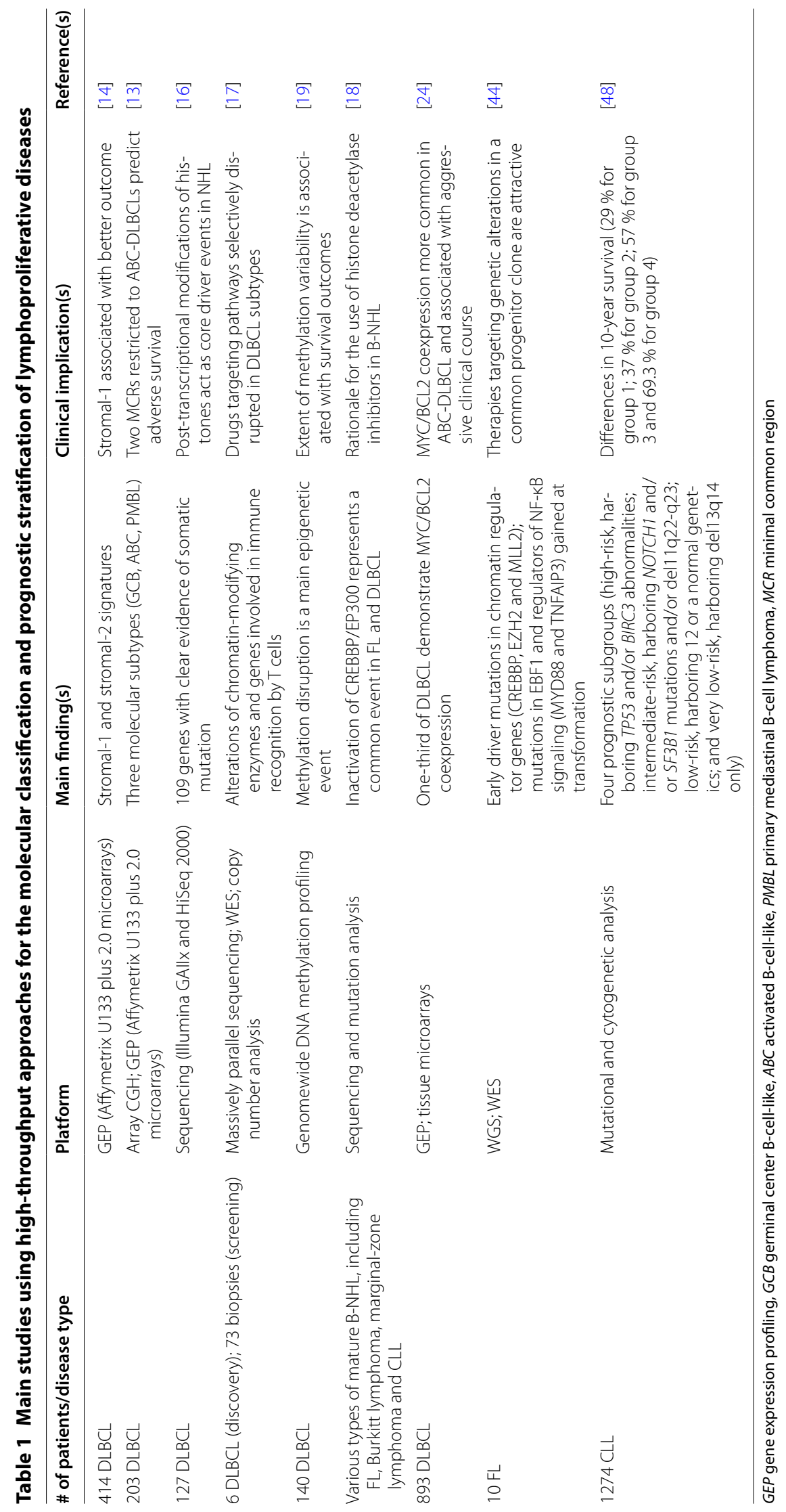


of the NF- $\mathrm{kB}$ pathway are constitutively activated in ABC-DLBCLs $[25,26]$. The activation of NF- $\mathrm{kB}$ in ABCDLBCLs consists of an amplification of upstream oncogenic signalling, since it creates multiple feed-forward and feed-back signaling loops through transcriptional activation of target genes required for survival [27]. ABCDLBCLs with an activated JAK/STAT signaling have a higher NF- $k B$ target gene expression [12]. Interference with NF- $\mathrm{KB}$ activity induces cell cycle arrest and triggers apoptosis. In this respect, small-molecule inhibitors blocking the degradation of the NF- $\mathrm{kB}$ inhibitor $\mathrm{I} \kappa \mathrm{B} \alpha$ or the activation of IKK are reportedly toxic for $\mathrm{ABC}$ DLBCLs [28, 29].

The transcription factor IRF4 is, among others, a key player insofar it supports the survival of ABC-DLBCLs and, together with BAFF, is required downstream of $B C R$ signaling to promote transcriptional programs during germinal centre formation [30].

B lymphocytes express a surface membrane B-cell receptor (BCR), which comprises a canonical immunoglobulin (heavy and light chains) coupled to a heterodimer $\mathrm{CD} 79 \mathrm{a} / \mathrm{CD} 79 \mathrm{~b}(\operatorname{Ig} \alpha / \operatorname{Ig} \beta)$, which is required for plasma membrane expression, intracellular trafficking and signal transduction. A distinctive characteristic of the BCR is that ABC-DLBCLs often express IgM-BCR on their surface, whereas GCB-DLBCLs typically show IgGBCR switching [31]. There are inactivating events that specifically occur in the productive (in-frame) IgH allele in ABC-DLBCLs, whereas the non-productive (out of frame) IgH alleles switch to a different isotype [32]. This leads to a selective pressure on ABC-DLBCLs to maintain an IgM isotype in the IgM-BCR. The IgM- and IgG$\mathrm{BCR}$ induce qualitatively different signaling cascades and, ultimately, transcriptional programs.

The CARD11 cytoplasmic scaffolding protein is a bona fide oncogenic protein that, when mutated, contributes to constitutive activation of NF- $\mathrm{kB}$ and enhanced NF- $\mathrm{kB}$ activity upon antigen-receptor stimulation [33]. The CARD11 protein is part of the so-called "CBM-complex" that is critical to the activation of the classical NF- $\mathrm{kB}$ pathway downstream of $\mathrm{B}$ - and T-cell antigen receptors [34]. The CBM complex mediates signaling from BCR to NF- $\mathrm{kB}$. The Bruton tyrosine-kinase (Btk) is critical for the survival of $A B C$ cell lines with wild-type CARD11, but not GCB lines or ABC lines with mutated CARD11 [35]. Ibrutinib is a novel inhibitor of Btk that has significant antitumor activity towards relapsed and refractory CLL, mantle-cell lymphoma and is currently in clinical trials for DLBCL $[36,37]$. It is an irreversible inhibitor that forms a covalent bond with cysteine 481 adjacent to the active site of Btk, and has a high degree of specificity and potency. The better clinical response of $A B C$ DLBCLs to ibrutinib compared with GCB-DLBCLs supports the hypothesis that ABC-DLBCLs, but not GCB-DLBCLs, rely on the chronic activation of BCR signalling [38].

Another mechanism leading to the BCR-independent activation of the NF- $\kappa B$ pathway is mediated by the adapter MyD88, which is an inducer of NF- $\mathrm{kB}$ activity upon Toll-like receptor (TLR) stimulation of immune cells [39]. Mutant MYD88 isoforms, as well as ectopic expression of MyD88 mutants in heterologous cells, are potent activators of NF-kB [39].

Bortezomib is an NF- $\mathrm{KB}$ targeting drug that blocks the degradation of IкB $\alpha$ and has shown to benefit patients with relapsed ABC-DLBCLs, when combined with doseadjusted EPOCH-R chemotherapy [40].

\section{Use of high-throughput approaches to identify molecular targets in $\mathrm{FL}$}

FLs have features of germinal center B cells and are currently considered incurable diseases when treated with conventional chemotherapy or immunotherapy. Understanding the evolution dynamics of FL clones is crucial to monitor disease progression and to effectively target FLs. Whole-genome and transcriptome sequencing of $B C L 2$ translocation-positive FLs in comparison with IG-MYC translocation-positive Burkitt lymphoma and normal germinal center B-cell samples showed differential methylation of intragenic regions that strongly correlated with expression of genes active in germinal center dark-zone and light-zone B cells [41]. These data point to a close connection between somatic mutation, DNA methylation and transcriptional control in B-cell pathways in FLs and other germinal center B-cell lymphomas.

Genetic profiling of serial FL biopsies suggests that FLs may derive by divergent evolution from a common ancestor cell. The pattern of somatic hypermutation of the $I g$ gene provides useful information about B-cell clonal evolution within the germinal center and also allows to discriminate $B$ cells that entered the germinal center and display features of ancestor cells from B cells that continue to re-circulate across different lymphoid organs. The pattern of somatic hypermutation of the heavy chain of the immunoglobulin gene $(I g H-V H)$ was examined using the GS-FLX Titanium sequencing platform in flow-sorted B cells of various differentiation stage, obtained from lymph node biopsies of FL patients with various patterns of evolution [42]. The authors reported a high level of clonality, with hundreds of distinct tumor subclones being detected in different cell subpopulations from the same patient sample. By using a lineage trees analysis, the oligoclonal FL population in all cases was shown to reside in an intermediate stage of maturation that maintains the capacity to undergo SHM, but is unable to further differentiate. 
Intraclonal heterogeneity in FLs is supported by studies of SHM caused by AID in IGH. Aberrant SHM is defined as AID activity outside of the $I G$ loci and mainly targets noncoding regions, inducing "passenger" mutations, but also having the potential to generate rare "driver" mutations. Ultradeep sequencing $(>20,000-$ fold coverage) has been used to define the quantitative relationship between SHM and aberrant SHM on IGH ( 1650 nt) and nine other noncoding regions potentially targeted by AID [43]. Single-nucleotide variants (SNVs) could be detected in 12/12 FL specimens. The aberrant SHM SNVs were associated with AID motifs. The relative number of SNVs with variable allele frequency $<5 \%$ varied with clinical grade, suggesting that tumor heterogeneity based on aberrant SHM may serve as a clinically relevant parameter.

Another study of WGS or WES followed by deep sequencing of 28 genes led to the identification of recurrent FL mutations in linker histone, JAK-STAT signaling, NF- $\mathrm{kB}$ signaling and B-cell developmental genes [44]. Longitudinal analyses allowed the detection of early driver mutations in chromatin regulator genes, whereas mutations in EBF1 and regulators of NF- $\mathrm{kB}$ signaling were gained at transformation. The remarkable intratumoral diversity within FLs is supported by studies showing a clonal hierarchy when comparing diagnosis and relapse tumor pairs, resolved using immunoglobulin somatic mutations and IGH-BCL2 translocations [45]. Using this approach, early versus late genetic events were clearly distinguished during lymphomagenesis. More specifically, IGH-BCL2 translocations and CREBBP mutations may be early events, whereas MLL2 and TNFRSF14 mutations may represent late events during FL evolution.

\section{Use of high-throughput approaches to identify molecular targets in CLL}

CLL is a common B-cell malignancy characterized by a highly variable clinical course. A "watch-and-wait" therapeutic approach has long been advocated for patients without symptomatic disease, with frontline chemotherapy being a conventional choice for patients requiring treatment. More recently, new drugs have been approved for the treatment of patients with CLL, including novel CD20-targeting antibody obinutuzumab, PI3-kinase inhibitor idelalisib, and irreversible inhibitor of Bruton tyrosine kinase ibrutinib [46]. Our understanding of CLL genetics has been furthered by large-scale studies of massively parallel sequencing, which have unveiled the genetic and epigenetic heterogeneity among patients, and within individual patient samples. The most recurrent lesions identified, i.e., deletions of chromosome 13q ( $55 \%$ of cases), 17p (7 \%), and 11q (6-18\%); and trisomy 12
(12-16 \%), reportedly contain putative CLL drivers, such as ATM and BIRC3 in 11q, TP53 in 17p, and miR-15a/16 encoded in an intron of DLEU2 in 13q [46]. Other clinically relevant gene mutations in CLL involve NOTCH1, SF3B1, and MYD88 [47]. It has been shown that addition of integrated mutational and cytogenetic information can improve prediction of OS in patients with CLL compared with cytogenetics alone [48].

The SHM status of the clonotypic rearranged immunoglobulin (Ig) heavy chain variable region (IGHV) gene is strongly associated with patient outcome [49]. Recently, the complete haplotype DNA sequence of the human Ig heavy-chain variable, diversity and joining genes was described. The authors also identified four large germline copy-number variants (CNVs) and eight CNV-containing haplotypes in addition to previously unmapped IGHV genes [50]. The international ImMunoGeneTics information system (IMGT ${ }^{\circledR}$ ) has been enriched with these novel IGHV genes and alleles and the new sequences have been incorporated into a database and into reference directories [51]. The tool used to analyze the rearranged IGH sequences was the IMGT/highV-QUEST portal for NGS Ig and T-cell receptor sequences [52]. This is a highthroughput version of the IMGT/V-QUEST that meets the needs of deep-sequencing data analysis coming from NGS platforms, allowing for the simultaneous analysis of thousands of Ig and T-cell receptor sequences in a single run.

Genome-wide chromatin accessibility maps for CLL have been measured by the assay for transposase-accessible chromatin using sequencing (ATAC-seq) [53]. Significant differences in chromatin accessibility were shown for regions in the vicinity of genes that were identified as differentially expressed between $I G H V$-mutated and $I G H V$-unmutated CLL, allowing partial separation of two disease subtypes. IGHV mutation status could be predicted by the chromatin profiles and gene regulatory networks inferred for $I G H V$-mutated vs. $I G H V$-unmutated samples. This chromatic profiling assays should be straightforward to use in a clinical sequencing laboratory, allowing deeper insights into chromatic regulation in CLL as well as detection of biomarkers for stratified cancer therapy.

The presence of uracil into the DNA sequences was, until recently, considered the result of a misincorporation of dUMP during DNA replication and of spontaneous deamination of DNA cytosine. AID belongs to the family of APOBEC cytosine deaminases and represents a third source of uracil within the DNA sequence [54]. The DNA cytosine deamination by APOBECfamily enzymes is a natural event in both adaptive and innate immune responses through targeted deamination of Ig genes by AID and deamination of viral DNA 
by APOBEC enzymes, respectively [55]. Interestingly, recent data demonstrate that B-cell lymphoma cell lines contain several-fold increased levels of genomic uracil compared to normal human lymphocytes and non-lymphoma cell lines. Moreover, genomic uracil content correlated with AID protein expression and not with other APOBEC enzymes [54]. Analysis of exome sequencing data from lymphomas and CLL unravelled that these lymphoid malignancies may carry a distinct AID-hotspot mutational signature in kataegis regions, i.e., localized hypermutations in small regions that are also associated with genomic rearrangements [56]. Thus, AID-induced genomic uracil formation may be implicated in the development of localized hypermutation in B-cell malignancies [54].

A significant application of NGS allowed the assessment of the subclonal development based on mutations in the $I G H V-D-J$ signature sequence in the dominant CLL clone [57]. This approach consistently evidenced, the occurrence of APOBEC and AID targeted mutations.

WGS studies in low-risk CLL patients with chromosome 13q deletion or normal cytogenetics showed that mutations in known CLL drivers are observed in only $33 \%$ of this patient cohort, and are associated with normal cytogenetics and unmutated IGHV [58]. The IGLL5 gene, which is homologous to IGLL1 (lambda5) and is critical for B-cell development, was the most commonly mutated gene and the mutational pattern suggested the potential involvement of AID activity. Three mutational signatures were identified, including two distinct AID processes (canonical AID and noncanonical-AID) that represent a greater fraction of mutational activity in mutated IGHV cases, and an ageing-related signature. The clonal or subclonal nature of a mutation was used to infer the time of occurrence of that mutation in relation to initial malignant transformation, the prediction being that clonal mutations occur earlier. Using this approach, noncanonical-AID-associated mutations were shown to occur in tumour evolution, whereas the ageing signature activity was enriched in patients with late-onset disease and enriched in subclonal mutations, which occur later [58].

The efficacy of WES approach in the discovery of driver mutations in CLL is further confirmed by the finding that 18 novel driver mutations were unveiled only with the use of this technique. These novel driver mutations hit histone proteins (HIST1H1D; HIST1HIC) involved in resistance to lenalidomide in multiple myeloma (IKZF3), nuclear RNA export factor 1 (NXF1) and proteins also mutated in acute myeloid leukemia and multiple myeloma (ASXL1; TRAF3) [59]. RNA sequencing (RNAseq) approaches were next used to validate the novel driver mutations emerged from WES analysis.
Proteomic analysis also represents a useful approach to the molecular classification of CLL. The mutational status of the $I G H V$ genes defines two clinically distinct disease forms, known as mutated CLL (M-CLL) and unmutated CLL (UM-CLL). Patients with M-CLL usually have a favorable outcome, whereas patients with UM-CLL develop progressive disease and have shorter survival [60]. Quantitative proteomic analysis through the use of the iTRAQ-MS platform allowed the identification of two subsets of CLL clearly reflecting the IGHV mutational status. Specifically, 274 proteins were found to be differentially expressed when comparing UM-CLL and M-CLL samples. Proteomic analysis also allowed the establishment of a novel correlation between lymphadenopathy and UM-CLL, leading to the hypothesis that retention of malignant $B$ cells in lymph nodes of UMCLLs may underpin the development of lymphadenopathy [61].

Double $I G H V$ gene rearrangements have been shown to correlate with patient prognosis in CLL [62]. Although differences in survival were not detected between patients with single or double $I G H V$ chain rearrangements, the presence of at least one mutated IGHV gene conferred a better prognosis to patients with double $I G V H$ rearrangements, suggesting that patients with double $I G H V$ rearrangements with at least one mutated $I G H V$ should be offered the same treatment intensity as those with a mutated rearrangement [62].

CLL is sensitive to immune system attack and is amenable to immune interventions, including the adoptive transfer of genetically modified T cells [63]. Rajasagi and co-workers [64] used WES and WGS to identify neoantigens presented by patient-specific HLA alleles. Neoantigens are generated from peptides encoded by gene alterations that are exclusively present in the tumor but not in normal tissues, and qualify as attractive targets for anti-tumor vaccination strategies. Using samples from 91 patients with CLL, 22 mutated binding peptides were predicted. In addition, HLA binding was experimentally confirmed for $55 \%$ of such peptides. Neoantigen-specific T-cell responses were also monitored in CLL patients in continuous complete remission for $>4$ years after reduced-intensity hematopoietic stem cell transplantation (HSCT). In the first patient, 25 missense mutations were identified by WES. Patient T cells were expanded using antigen-presenting cells and peptide pools from the candidate neoantigen. Based on IFN- $\gamma$ release, reactivity against the mutated peptides, but not against irrelevant peptides, was readily identified. In the second patient, 26 missense mutations were discovered and polyfunctional, highly avid memory $\mathrm{T}$-cell responses were measured in vitro. Using mutant-specific tetramers, neoantigenspecific $\mathrm{T}$ cells could be detected and tracked in vivo 
coinciding with the achievement of molecular remission after HSCT.

Chimeric antigen receptor (CAR)-modified $\mathrm{T}$ cells have recently generated enthusiasm as an immunotherapy approach for lymphoproliferative malignancies [65]. Whereas CAR $\mathrm{T}$ cells typically target highly expressed antigens, low antigen-expressing tumours may develop resistance to CAR T-cell therapy. Interestingly, epigenetic modulators were shown to increase the expression levels of tumour-associated antigens, thus rendering tumour targets highly susceptible to immune system attack. The hypomethylating agent 5-aza-2'-deoxycytidine (DAC) induces NY-ESO-1, a cancer-testis antigen, in multiple myeloma and breast cancer cells in a time- and dosedependent manner, with maximal effects being shown after incubation with $10 \mu \mathrm{M}$ DAC for $72 \mathrm{~h}$ [66]. Single NY-ESO-1 peptides presented in the context of HLA$\mathrm{A} * 0201$ molecules were increased by 100 and $50 \%$ on MCF7 and U266 cells, respectively. This was reflected into an increased lysis of tumour targets by HLAA*0201/NY-ESO-1 $1_{157-165}$ peptide-specific CAR CD8 ${ }^{+} \mathrm{T}$ cells [66]. A similar approach has been used to increase the intensity of other tumour-associated antigens, i.e., mucin-1 (MUC-1) and prostate stem cell antigen (PSCA) [67]. In mice, complete eradication of pancreatic tumours is not achieved irrespective of the simultaneous challenge of pancreatic tumour cells (CAPAN1) with CAR $\mathrm{T}$ cells targeting both MUC-1 and PSCA [67]. However, CAPAN1 cells expressing low levels of MUC-1 as a result of immune escape mechanisms can be re-sensitised to the killing effect of CAR T cells after 4-day treatment with decitabine. Overall, these studies suggest that hypoethylating agents could be of benefit in the context of CAR T-cell immunotherapy trials that target a variety of tumour types.

\section{Mutational burden and immune checkpoint inhibitors in lymphoid malignancies}

The use of immune checkpoint inhibitors for the treatment of haematological malignancies has raised considerable interest [68]. These agents do not target the cancer cells directly but rather enhance the cytotoxic activity of host $\mathrm{T}$ cells by blocking inhibitory signals from tumour cells. Cancer types harbouring more somatic mutations, i.e., lung cancer and melanoma, are expected to more frequently express neo-antigens that are recognized by autologous $\mathrm{T}$ cells, and should be more susceptible to checkpoint inhibitors [69]. It is presently unknown whether lymphoid malignancies may be less amenable to treatment with checkpoint inhibitors than solid tumours, based on the lower number of somatic mutations per megabase of coding DNA. Differences in response have been demonstrated across various haematological malignancies [70]. For instance, checkpoint inhibition is undoubtedly a promising strategy in classical Hodgkin lymphoma (HL). Recent analyses integrating high-resolution copy-number data and transcriptional profiles identified PD-L1 and PD-L2 as key targets of 9p24.1 amplification, which is a recurrent genetic abnormality in $\mathrm{HL}$ and may lead to overexpression of the PD-1 ligands on the Reed-Sternberg cell surface [71]. A recent study enrolled 23 patients with multiply relapsed or refractory HL, who received nivolumab (at a dose of $3 \mathrm{mg}$ per kilogram of body weight) every 2 weeks until they had a complete response, tumor progression, or excessive toxic effects [72]. An objective response was reported in $87 \%$ of patients, including $17 \%$ with a complete response and $70 \%$ with a partial response. The remaining $13 \%$ of patients had stable disease. Analyses of pretreatment tumor specimens from a subgroup of ten patients showed copy number gains in PD-L1 and PD-L2 as well as increased expression of these ligands [72].

Immune checkpoint blockade has been shown to exert therapeutic activity in at least FL and DLBCL. A phase $2 \mathrm{MD}$ Anderson Cancer Centre trial to investigate the activity of pidilizumab, a humanised anti-PD1 monoclonal antibody, with rituximab in 32 adult patients with relapsed FL showed objective responses in $66 \%$ of evaluable patients, including complete responses in $52 \%$ and partial responses in $14 \%$ of patients [73].

A phase Ib study in relapsed or refractory B-cell lymphoma, T-cell lymphoma, and multiple myeloma has shown the efficacy of the anti-PD-1 monoclonal antibody nivolumab, with 40,36, 15 and $40 \%$ objective response rates among patients with FL, DLBCL, mycosis fungoides, and peripheral T-cell lymphoma, respectively [74]. By contrast, tumour regression responses were not observed among patients with multiple myeloma, with the exception of one complete remission occurring after local radiation therapy. The reason for the lack of objective responses in MM, compared with other tumors, remain speculative and may be related to immunosuppressive circuits within the myeloma tumour microenvironment [75]. Also, differences in the composition and quality of the immune infiltrate may account for differences in clinical responses across the wide spectrum of lymphoid malignancies.

\section{The emerging role of epigenomics}

Epigenetics and epigenomics focus on the study of chromatin, i.e., the complex of DNA, proteins, and non-coding RNAs (ncRNAs) that form the structural matrix of a chromosome. The spectrum of epigenetic modifications that regulate chromatin encompasses DNA methylation, histone modifications, and ncRNAs [76]. Epigenetic changes are modifications that do not alter the genomic 
nucleotide sequence but influence gene regulation and expression [77]. Changes involving gene expression control through epigenetic modifications are increasingly recognized as major mechanisms involved in the development of a variety of diseases, including cancer. These modifications can be reverted through pharmacologic treatment (such as small molecules, antibodies and inhibitors) and, therefore, identification of groups of patients with specific epigenomic alterations may lead to therapeutic benefits [78]. Furthermore, seven among the top ten leading causes of death listed by the World Health Organization in 2010 were diseases with an epigenetic and epigenomic component, underlying the need for boosting research efforts in this field [79]. The most updated molecular techniques and related bioinformatics platforms used for the epigenomic profiling of patients are shown schematically in Fig. 1.

DNA methylation is a key epigenetic mechanism used by higher eukaryotes for gene expression regulation, imprinting and silencing of germline specific genes and repetitive elements [80]. Methylation patterns are stably maintained through somatic cell division and can be inherited across generations. Since perturbed methylation patterns are linked to pathologic conditions such as imprinting disorders and cancer, understanding how these patterns are established and maintained is of great importance [81].

It is increasingly recognized that the epigenomic profiling of patients represents a useful tool in the path towards personalized medicine [77]. A growing body of evidence suggests that different molecular approaches should be integrated in order to build a realistic profile of the disease status of patients. Among these advanced technologies, epigenomic profiling is becoming a fundamental tool to explore gene expression regulation. Dysfunctional methylation patterns, for instance, may become the signature of a specific cancer whereas overexpression of specific deacetylases can be univocally associated with distinct malignancies. As epigenetic therapies advance towards the clinic, genomic/epigenomic tools will support the identification of patients who will most likely benefit from hypomethylating drugs.

Very informative in this context is the National Institutes of Health (NIH) Epigenomics Roadmap, a bioinformatics platform prompted by the NIH Roadmap Epigenomics Mapping Consortium [82]. This consortium developed a panel of resources based on NGS technologies in order to map DNA methylation, histone modifications, chromatin accessibility and small RNA transcripts in stem cells and primary ex vivo tissues that represent normal counterparts of tissues frequently involved in human disease. The NIH Epigenomics roadmap provides not only updated databases with the epigenomes of human cell types, tissues or whole organs but also protocols and reference guidelines for the scientific community.

Aberrant DNA methylation pattern is considered a hallmark of cancer, and may contribute to lymphoma phenotypes and chemoresistance of cancer cells. Decitabine is a nucleoside analogue that, after being incorporated into DNA, inhibits DNA methyltransferases

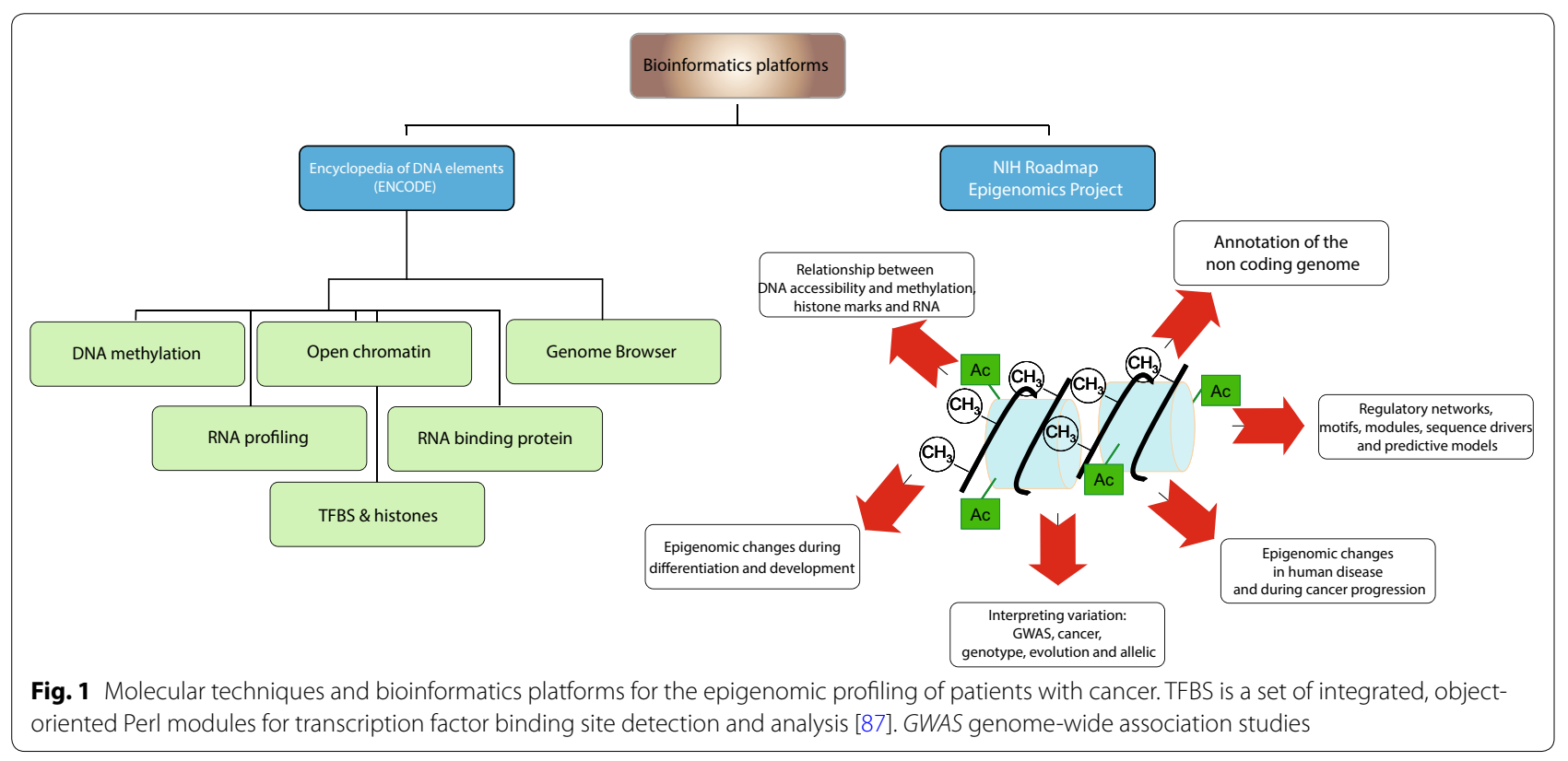


(DNMTs) and acts as an hypomethylating agent. Decitabine has been approved for the treatment of myelodysplastic syndromes. A recent phase I study aimed at targeting aberrant DNA methylation in relapsed/ refractory CLL and NHL by administering 1-3 cycles of decitabine [83]. Dose-limiting toxicity, consisting of myelosuppression and infectious complications, was documented in 2 of 4 patients with CLL and in 2 of 2 patients with NHL given decitabine at $15 \mathrm{mg} / \mathrm{m}^{2}$. Six patients received $10 \mathrm{mg} / \mathrm{m}^{2}$ decitabine for 10 days without DLTs and with no change in global DNA methylation or reexpression of methylated genes. When a 5-day decitabine schedule was evaluated, DLTs were observed in 2 of 6 CLL and 2 of 2 NHL patients, suggesting that dose escalation to levels associated with methylation changes may be difficult to achieve in patients.

The relevance of targeting DNMTs has also been explored in DLBCLs where chemoresistance is associated with aberrant DNA methylation programming [84]. Prolonged exposure to low-dose DNMT inhibitors reprogrammed chemoresistant cells and restored sensitivity to doxorubicin. A group of nine genes was found to be recurrently hypermethylated in chemoresistant DLBCL with SMAD1, a TGF- $\beta$ pathway transducer, being an important contributor. A subsequent phase I study evaluated the impact of azacitidine priming for 5 days, followed by R-CHOP chemo-immunotherapy, in 12 patients with newly diagnosed DLBCL. Eleven of 12 patients achieved a complete response and ten remained in remission at median follow-up of 13 months. Patient biopsies obtained before and after treatment confirmed an increase of SMAD1 mRNA and a decrease in SMAD1 methylation, as well as a remarkable increase in the fraction of dead cells in the post-azacitidine samples. This study suggests that DNMT inhibitors may play a role in priming high-risk DLBCLs before administration of chemotherapy.

Decitabine has also been used in combination with vorinostat, a small molecule that binds to and directly inhibits histone deacetylase, and is approved for the treatment of cutaneous T-cell lymphoma. This phase I study enrolled 43 patients with advanced solid tumors and NHL [85]. Both sequential and concurrent schedules were evaluated in nine different dose levels ( 6 sequential and 3 concurrent). The sequential schedule of decitabine $10 \mathrm{mg} / \mathrm{m}^{2} /$ day on days $1-5$ and vorinostat $200 \mathrm{mg}$ twice a day on days 6-12 qualified as the recommended phase II dose in this study. Disease stabilization for four cycles or more was observed in approximately $30 \%$ of evaluable patients, suggesting that the combination of decitabine with vorinostat is tolerable and shows activity in different tumor types.

\section{Therapeutic implications of genetic findings}

Recent insights into the molecular complexity of lymphoproliferative disorders are expected to herald breakthroughs in disease classification, prognostication and treatment. In addition to the major molecular designations of GCB and $\mathrm{ABC}$ subtypes, NGS technologies have unravelled the remarkable heterogeneity and complexity of DLBCL and identified molecular targets that may be selectively exploited for therapeutic benefit. Classifying NHLs based on cell-of-origin subtypes or presence of other molecular features portends important biologi$\mathrm{cal}$, prognostic and therapeutic implications. Agents targeting the oncogenic drivers of DLBCL subsets might expand the number of DLBCL patients achieving a cure, and are in various stages of investigation, as reviewed elsewhere $[15,28]$.

Molecular similarities between DLBCL and FL have provided insight into the potential of both NHL subtypes to respond to agents targeting common genetic alterations [86]. Despite differences in clinical behaviour, FL, similar to GCB-DLBCL, develops from GC B cells. Markers of prognostic significance may be relevant to both DLBCL and FL management [86].

Whole genome/exome sequencing has also been instrumental in disclosing the genetic landscape of CLL, revealing mutations of cancer-related genes and events involved in disease initiation and progression. BCR signalling is an attractive molecular target in patients with CLL, with the BTK inhibitor ibrutinib showing clinical activity $[36,47]$.

\section{Concluding remarks}

The landscape of genomic and epigenomic changes associated with tumor transformation is increasingly disclosed due to the availability of new sequencing technologies and bioinformatic tools. Our appreciation of the heterogeneity of lymphoid tumors has grown following the use of high-throughput approaches. The unprecedented possibilities offered by these technologies will allow researchers and clinicians to develop novel and more effective treatment approaches. As the path towards integrative medicine and personalized treatments progresses, knowledge sharing through informatics databases will accelerate the dissemination and validation of experimental findings.

\footnotetext{
Abbreviations

DLBCL: diffuse large B cell lymphoma; FL: follicular lymphoma; CLL: chronic lymphocytic leukemia; SLL: small lymphocytic lymphoma; SM: systems medicine; WGS: whole genome sequencing; WES: whole exome sequencing; NGS: next generation sequencing; GEP: gene expression profiling; DNMT: DNA methyltransferase; HSCT: hematopoietic stem cell transplantation; CHOP: cyclophosphamide, doxorubicin, vincristine, and prednisone.
} 


\section{Authors' contributions}

$R F, C A, A F, P F, S R$ and $A C$ mined the literature and contributed to manuscript writing. All authors read and approved the final manuscript.

\section{Authors' information}

C.A. is currently involved in the eTRIKS consortium (translational research information and knowledge management services), the Coordination action CASyM to implementat systems medicine across Europe, and also chairs the newly formed European Association of Systems Medicine (EASyM).

S.R. is a licensed hematologist with 25 years of clinical and laboratory experience in cell therapy, hematopoietic stem cell transplantation and cancer immunotherapy. He is currently the Professor of Cancer Immunotherapy at the John van Geest Cancer Research Centre, Nottingham Trent University, UK.

A.C. is Deputy Director of Governance at the Catholic University Medical School in Rome, Italy; Member of the European Association for Systems Medicine-EASyM; and Co-Founder and Vice-President of The Italian Society of Systems Medicine and Healthcare (ASSIMSS).

\begin{abstract}
Author details
1 Laboratory of Translational Research, IRCCS "Arcispedale S. Maria Nuova", Reggio Emilia, Italy. ${ }^{2}$ European Institute for Systems Biology and Medicine (EISBM), Paris, France. ${ }^{3}$ Division of Hematology, IRCCS "Arcispedale S. Maria Nuova", Reggio Emilia, Italy. ${ }^{4}$ Division of Translational Medicine, Sidra Medica and Research Centre, Doha, Qatar. ${ }^{5}$ John van Geest Cancer Research Centre, College of Science and Technology, Nottingham Trent University, Clifton Campus, Nottingham NG11 8NS, UK. ${ }^{6}$ Clinical Governance and International Research Activities, Fondazione Policlinico Gemelli, Rome, Italy. ${ }^{7}$ Division of Thoracic Surgery, Università Cattolica del Sacro Cuore, Rome, Italy. ${ }^{8}$ European Association of Systems Medicine, Aachen, Germany.
\end{abstract}

\section{Competing interests}

The authors declare that they have no competing interests.

\section{Funding}

This work was partly supported by Qatar National Research Fund (QNRF) through a National Priorities Research Programme award to S.R. (NPRP8-2297-3-494).

\section{Received: 14 June 2016 Accepted: 18 August 2016}

Published online: 01 September 2016

\section{References}

1. Cesario A, Auffray C, Russo P, Hood L. P4 medicine needs P4 education. Curr Pharm Des. 2014;20:6071-2.

2. Cesario A, Auffray C, Agusti A, Apolone G, Balling R, Barbanti P, Bellia A Boccia S, Bousquet J, Cardaci V, et al. A systems medicine clinical platform for understanding and managing non-communicable diseases. Curr Pharm Des. 2014;20:5945-56.

3. Watt CD, Roullet MR, Bagg A. Application of molecular genetics to the diagnosis and classification of hematologic neoplasms. In: Orazi A, Weiss LM, Foucar K, Knowles DM, editors. Neoplastic hematopathology. Alphen aan den Rijn: Wolters Kluwer; 2014. p. 194-231.

4. Sant M, Minicozzi P, Mounier M, Anderson LA, Brenner $H$, Holleczek B, Marcos-Gragera R, Maynadie M, Monnereau A, Osca-Gelis G, et al. Survival for haematological malignancies in Europe between 1997 and 2008 by region and age: results of EUROCARE-5, a population-based study. Lancet Oncol. 2014;15:931-42.

5. Cancer Stat Fact Sheets. http://seer.cancer.gov/statfacts/html/nhl.html. Accessed 15 Aug 2016.

6. Alt FW, Zhang Y, Meng FL, Guo C, Schwer B. Mechanisms of programmed DNA lesions and genomic instability in the immune system. Cell. 2013;152:417-29.

7. Li Y, Wang Y, Wang Z, Yi D, Ma S. Racial differences in three major NHL subtypes: descriptive epidemiology. Cancer Epidemiol. 2014;39:8-13.

8. Campo E, Swerdlow SH, Harris NL, Pileri S, Stein H, Jaffe ES. The 2008 WHO classification of lymphoid neoplasms and beyond: evolving concepts and practical applications. Blood. 2011;117:5019-32.
9. Swerdlow SH, Campo E, Lee Harris N, Jaffe ES, Pileri S, Stein H, Thiele J, Vardiman JW. WHO classification of tumors of haematopoietic and lymphoid tissues. Lyon: International Agency for Research on Cancer (IARC); 2008.

10. Pfreundschuh M, Schubert J, Ziepert M, Schmits R, Mohren M, Lengfelder E, Reiser M, Nickenig C, Clemens M, Peter N, et al. Six versus eight cycles of bi-weekly CHOP-14 with or without rituximab in elderly patients with aggressive CD20+ B-cell lymphomas: a randomised controlled trial (RICOVER-60). Lancet Oncol. 2008;9:105-16.

11. Pfreundschuh $M$, Trumper $L$, Osterborg A, Pettengell $R$, Trneny M, Imrie K, Ma D, Gill D, Walewski J, Zinzani PL, et al. CHOP-like chemotherapy plus rituximab versus $\mathrm{CHOP}$-like chemotherapy alone in young patients with good-prognosis diffuse large-B-cell lymphoma: a randomised controlled trial by the MabThera International Trial (MInT) Group. Lancet Oncol. 2006;7:379-91.

12. Young RM, Shaffer AL 3rd, Phelan JD, Staudt LM. B-cell receptor signaling in diffuse large B-cell lymphoma. Semin Hematol. 2015;52:77-85.

13. Lenz G, Wright GW, Emre NC, Kohlhammer H, Dave SS, Davis RE, Carty S, Lam LT, Shaffer AL, Xiao W, et al. Molecular subtypes of diffuse large B-cell lymphoma arise by distinct genetic pathways. Proc Natl Acad Sci USA. 2008:105:13520-5.

14. Lenz G, Wright G, Dave SS, Xiao W, Powell J, Zhao H, Xu W, Tan B, Goldschmidt N, labal J, et al. Stromal gene signatures in large-B-cell lymphomas. N Engl J Med. 2008;359:2313-23.

15. Sehn LH, Gascoyne RD. Diffuse large B-cell lymphoma: optimizing outcome in the context of clinical and biologic heterogeneity. Blood. 2015;125:22-32.

16. Morin RD, Mendez-Lago M, Mungall AJ, Goya R, Mungall KL, Corbett RD, Johnson NA, Severson TM, Chiu R, Field M, et al. Frequent mutation of histone-modifying genes in non-Hodgkin lymphoma. Nature. 2011;476:298-303.

17. Pasqualucci L, Trifonov V, Fabbri G, Ma J, Rossi D, Chiarenza A, Wells VA, Grunn A, Messina M, Elliot O, et al. Analysis of the coding genome of diffuse large B-cell lymphoma. Nat Genet. 2011;43:830-7.

18. Pasqualucci L, Dominguez-Sola D, Chiarenza A, Fabbri G, Grunn A, Trifonov V, Kasper LH, Lerach S, Tang H, Ma J, et al. Inactivating mutations of acetyltransferase genes in B-cell lymphoma. Nature. 2011;471:189-95.

19. Chambwe N, Kormaksson M, Geng H, De S, Michor F, Johnson NA, Morin RD, Scott DW, Godley LA, Gascoyne RD, et al. Variability in DNA methylation defines novel epigenetic subgroups of DLBCL associated with different clinical outcomes. Blood. 2014;123:1699-708.

20. Barrans S, Crouch S, Smith A, Turner K, Owen R, Patmore R, Roman E, Jack A. Rearrangement of MYC is associated with poor prognosis in patients with diffuse large B-cell lymphoma treated in the era of rituximab. J Clin Oncol. 2010:28:3360-5.

21. Savage KJ, Johnson NA, Ben-Neriah S, Connors JM, Sehn LH, Farinha P, Horsman DE, Gascoyne RD. MYC gene rearrangements are associated with a poor prognosis in diffuse large B-cell lymphoma patients treated with R-CHOP chemotherapy. Blood. 2009;114:3533-7.

22. Friedberg JW. Double-hit diffuse large B-cell lymphoma. J Clin Oncol. 2012;30:3439-43.

23. Johnson NA, Slack GW, Savage KJ, Connors JM, Ben-Neriah S, Rogic S, Scott DW, Tan KL, Steidl C, Sehn LH, et al. Concurrent expression of MYC and BCL2 in diffuse large B-cell lymphoma treated with rituximab plus cyclophosphamide, doxorubicin, vincristine, and prednisone. J Clin Oncol. 2012:30:3452-9.

24. Hu S, Xu-Monette ZY, Tzankov A, Green T, Wu L, Balasubramanyam A, Liu WM, Visco C, Li Y, Miranda RN, et al. MYC/BCL2 protein coexpression contributes to the inferior survival of activated B-cell subtype of diffuse large B-cell lymphoma and demonstrates high-risk gene expression signatures: a report from The International DLBCL Rituximab-CHOP Consortium Program. Blood. 2013;121:4021-31.

25. Ben-Neriah $Y$, Karin M. Inflammation meets cancer, with NF-KB as the matchmaker. Nat Immunol. 2011;12:715-23.

26. Davis RE, Brown KD, Siebenlist U, Staudt LM. Constitutive nuclear factor $\mathrm{KB}$ activity is required for survival of activated $B$ cell-like diffuse large $B$ cell Iymphoma cells. J Exp Med. 2001;194:1861-74.

27. Shaffer AL 3rd, Young RM, Staudt LM. Pathogenesis of human B cell lymphomas. Annu Rev Immunol. 2012;30:565-610.

28. Roschewski M, Staudt LM, Wilson WH. Diffuse large B-cell lymphomatreatment approaches in the molecular era. Nat Rev Clin Oncol. 2014:11:12-23. 
29. Lam LT, Davis RE, Pierce J, Hepperle M, Xu Y, Hottelet M, Nong Y, Wen D, Adams J, Dang L, Staudt LM. Small molecule inhibitors of IKB kinase are selectively toxic for subgroups of diffuse large B-cell lymphoma defined by gene expression profiling. Clin Cancer Res. 2005;11:28-40.

30. Ochiai K, Katoh Y, Ikura T, Hoshikawa Y, Noda T, Karasuyama H, Tashiro S, Muto A, Igarashi K. Plasmacytic transcription factor Blimp-1 is repressed by Bach2 in B cells. J Biol Chem. 2006;281:38226-34.

31. Lenz G, Nagel I, Siebert R, Roschke AV, Sanger W, Wright GW, Dave SS, Tan B, Zhao H, Rosenwald A, et al. Aberrant immunoglobulin class switch recombination and switch translocations in activated $B$ cell-like diffuse large B cell lymphoma. J Exp Med. 2007;204:633-43.

32. Ruminy P, Etancelin P, Couronne L, Parmentier F, Rainville V, Mareschal S, Bohers $E$, Burgot $C$, Cornic $M$, Bertrand $P$, et al. The isotype of the BCR as a surrogate for the GCB and $A B C$ molecular subtypes in diffuse large B-cell Iymphoma. Leukemia. 2011;25:681-8.

33. Lenz G, Davis RE, Ngo VN, Lam L, George TC, Wright GW, Dave SS, Zhao H, Xu W, Rosenwald A, et al. Oncogenic CARD11 mutations in human diffuse large B cell lymphoma. Science. 2008;319:1676-9.

34. Thome M, Charton JE, Pelzer C, Hailfinger S. Antigen receptor signaling to NF-kB via CARMA1, BCL10, and MALT1. Cold Spring Harb Perspect Biol. 2010;2:a003004

35. Davis RE, Ngo VN, Lenz G, Tolar P, Young RM, Romesser PB, Kohlhammer H, Lamy L, Zhao H, Yang Y, et al. Chronic active B-cell-receptor signalling in diffuse large B-cell lymphoma. Nature. 2010;463:88-92.

36. Aalipour A, Advani RH. Bruton tyrosine kinase inhibitors: a promising novel targeted treatment for B cell lymphomas. Br J Haematol. 2013;163:436-43.

37. Advani RH, Buggy JJ, Sharman JP, Smith SM, Boyd TE, Grant B, Kolibaba KS, Furman RR, Rodriguez S, Chang BY, et al. Bruton tyrosine kinase inhibitor ibrutinib (PCl-32765) has significant activity in patients with relapsed/ refractory B-cell malignancies. J Clin Oncol. 2013;31:88-94.

38. Wilson WH, Gerecitano J, Goy A, et al. The Bruton's tyrosine kinase (BTK) inhibitor, ibrutinib (PCI-32765), has preferential activity in the $A B C$ subtype of relapsed/refractory de novo diffuse large B-cell lymphoma (DLBCL): interim results of a multicenter, open-label, phase 2 study. In: ASH, editor. 54th ASH Annual Meeting and Exposition; Atlanta, USA. 2012.

39. Ngo VN, Young RM, Schmitz R, Jhavar S, Xiao W, Lim KH, Kohlhammer H, Xu W, Yang Y, Zhao H, et al. Oncogenically active MYD88 mutations in human lymphoma. Nature. 2011;470:115-9.

40. Dunleavy K, Pittaluga S, Czuczman MS, Dave SS, Wright G, Grant N, Shovlin M, Jaffe ES, Janik JE, Staudt LM, Wilson WH. Differential efficacy of bortezomib plus chemotherapy within molecular subtypes of diffuse large B-cell lymphoma. Blood. 2009;113:6069-76.

41. Kretzmer H, Bernhart SH, Wang W, Haake A, Weniger MA, Bergmann AK, Betts MJ, Carrillo-de-Santa-Pau E, Doose G, Gutwein J, et al. DNA methylome analysis in Burkitt and follicular lymphomas identifies differentially methylated regions linked to somatic mutation and transcriptional control. Nat Genet. 2015;47:1316-25.

42. Carlotti E, Wrench D, Rosignoli G, Marzec J, Sangaralingam A, Hazanov L, Michaeli M, Hallam S, Chaplin T, labal S, et al. High throughput sequencing analysis of the immunoglobulin heavy chain gene from flow-sorted B cell sub-populations define the dynamics of follicular lymphoma clonal evolution. PLoS One. 2015:10:e0134833.

43. Spence JM, Abumoussa A, Spence JP, Burack WR. Intraclonal diversity in follicular lymphoma analyzed by quantitative ultradeep sequencing of noncoding regions. J Immunol. 2014;193:4888-94.

44. Okosun J, Bodor C, Wang J, Araf S, Yang CY, Pan C, Boller S, Cittaro D, Bozek M, lqbal S, et al. Integrated genomic analysis identifies recurrent mutations and evolution patterns driving the initiation and progression of follicular lymphoma. Nat Genet. 2014;46:176-81.

45. Green MR, Gentles AJ, Nair RV, Irish JM, Kihira S, Liu CL, Kela I, Hopmans ES, Myklebust JH, Ji H, et al. Hierarchy in somatic mutations arising during genomic evolution and progression of follicular lymphoma. Blood. 2013;121:1604-11.

46. Guieze R, Wu CJ. Genomic and epigenomic heterogeneity in chronic lymphocytic leukemia. Blood. 2015;126:445-53.

47. Rossi D, Ciardullo C, Spina V, Gaidano G. Molecular bases of chronic lymphocytic leukemia in light of new treatments. Immunol Lett. 2013;155:51-5.

48. Rossi D, Rasi S, Spina V, Bruscaggin A, Monti S, Ciardullo C, Deambrogi C, Khiabanian H, Serra R, Bertoni F, et al. Integrated mutational and cytogenetic analysis identifies new prognostic subgroups in chronic lymphocytic leukemia. Blood. 2013;121:1403-12.

49. Xochelli A, Agathangelidis A, Kavakiotis I, Minga E, Sutton LA, Baliakas P, Chouvarda I, Giudicelli V, Vlahavas I, Maglaveras N, et al. Immunoglobulin heavy variable (IGHV) genes and alleles: new entities, new names and implications for research and prognostication in chronic lymphocytic leukaemia. Immunogenetics. 2015;67:61-6.

50. Watson CT, Steinberg KM, Huddleston J, Warren RL, Malig M, Schein J, Willsey AJ, Joy JB, Scott JK, Graves TA, et al. Complete haplotype sequence of the human immunoglobulin heavy-chain variable, diversity, and joining genes and characterization of allelic and copy-number variation. Am J Hum Genet. 2013;92:530-46.

51. Lefranc MP. Immunoglobulin and T cell receptor genes: IMGT((R)) and the birth and rise of immunoinformatics. Front Immunol. 2014;5:22.

52. Alamyar E, Duroux P, Lefranc MP, Giudicelli V. IMGT((R)) tools for the nucleotide analysis of immunoglobulin (IG) and $T$ cell receptor (TR) V-(D)-J repertoires, polymorphisms, and IG mutations: IMGTN-QUEST and IMGT/HighV-QUEST for NGS. Methods Mol Biol. 2012:882:569-604.

53. Rendeiro AF, Schmidl C, Strefford JC, Walewska R, Davis Z, Farlik M, Oscier D, Bock C. Chromatin accessibility maps of chronic lymphocytic leukaemia identify subtype-specific epigenome signatures and transcription regulatory networks. Nat Commun. 2016;7:11938.

54. Pettersen HS, Galashevskaya A, Doseth B, Sousa MM, Sarno A, Visnes T, Aas PA, Liabakk NB, Slupphaug G, Saetrom P, et al. AID expression in B-cell lymphomas causes accumulation of genomic uracil and a distinct AID mutational signature. DNA Repair. 2015:25:60-71.

55. Conticello SG. The AID/APOBEC family of nucleic acid mutators. Genome Biol. 2008;9:229

56. Qian J, Wang Q, Dose M, Pruett N, Kieffer-Kwon KR, Resch W, Liang G, Tang Z, Mathe E, Benner C, et al. B cell super-enhancers and regulatory clusters recruit AID tumorigenic activity. Cell. 2014;159:1524-37.

57. Chu CC, Pattern PE, MacCarthy T, Yuan C, Yan XJ, Barrientos JC, Kolitz JE, Allen SL, Rai KR, Chiorazzi N. IGHV-D-J ultra-deep sequencing reveals APOBEC and AID targeted mutations during clonal evolution of CLL in a xenograft mouse model. In: 56th American society of hematology annual meeting, San Francisco, CA; 2014

58. Kasar S, Kim J, Improgo R, Tiao G, Polak P, Haradhvala N, Lawrence MS, Kiezun A, Fernandes SM, Bahl S, et al. Whole-genome sequencing reveals activation-induced cytidine deaminase signatures during indolent chronic lymphocytic leukaemia evolution. Nat Commun. 2015;6:8866.

59. Landau D, Stewart C, Reiter JG, et al. Novel putative driver gene mutations in chronic lymphocytic leukemia (CLL): results from a combined analysis of whole-exome sequencing of 262 primary CLL samples. In: 56th American society of hematology annual meeting; San Francisco, CA; 2014

60. Eagle G, Jenkins RE, Till KJ, et al. Total proteome analysis identifies migration defects as a major pathogenetic factor in IGHV-unmutated chronic lymphocytic leukemia. In: 56th American Society of Hematology Annual Meeting; San Francisco, CA; 2014.

61. Eagle GL, Zhuang J, Jenkins RE, Till KJ, Jithesh PV, Lin K, Johnson GG, Oates M, Park K, Kitteringham NR, Pettitt AR. Total proteome analysis identifies migration defects as a major pathogenetic factor in immunoglobulin heavy chain variable region (IGHV)-unmutated chronic lymphocytic leukemia. Mol Cell Proteom. 2015;14:933-45.

62. Heyman B, Volkheimer AD, Weinberg JB. Double IGHV DNA gene rearrangements in CLL: influence of mixed-mutated and -unmutated rearrangements on outcomes in CLL. Blood Cancer J. 2016;6:e440.

63. Porter DL, Hwang WT, Frey NV, Lacey SF, Shaw PA, Loren AW, Bagg A, Marcucci KT, Shen A, Gonzalez V, et al. Chimeric antigen receptor T cells persist and induce sustained remissions in relapsed refractory chronic lymphocytic leukemia. Sci Transl Med. 2015;7:303ra139.

64. Rajasagi M, Shukla SA, Fritsch EF, Keskin DB, DeLuca D, Carmona E, Zhang W, Sougnez C, Cibulskis K, Sidney J, et al. Systematic identification of personal tumor-specific neoantigens in chronic lymphocytic leukemia. Blood. 2014;124:453-62.

65. Maus MV, Grupp SA, Porter DL, June $\mathrm{CH}$. Antibody-modified T cells: CARs take the front seat for hematologic malignancies. Blood. 2014;123:2625-35.

66. Klar AS, Gopinadh J, Kleber S, Wadle A, Renner C. Treatment with 5-aza2'-deoxycytidine induces expression of NY-ESO-1 and facilitates cytotoxic T lymphocyte-mediated tumor cell killing. PLoS One. 2015;10:e0139221. 
67. Anurathapan U, Chan RC, Hindi HF, Mucharla R, Bajgain P, Hayes BC, Fisher WE, Heslop HE, Rooney CM, Brenner MK, et al. Kinetics of tumor destruction by chimeric antigen receptor-modified T cells. Mol Ther. 2014;22:623-33.

68. Thanarajasingam G, Thanarajasingam U, Ansell SM. Immune checkpoint blockade in lymphoid malignancies. FEBS J. 2016;283:2233-44.

69. Schumacher TN, Schreiber RD. Neoantigens in cancer immunotherapy. Science. 2015;348:69-74.

70. Armand P. Immune checkpoint blockade in hematologic malignancies. Blood. 2015;125:3393-400.

71. Green MR, Monti S, Rodig SJ, Juszczynski P, Currie T, O'Donnell E, Chapuy B, Takeyama K, Neuberg D, Golub TR, et al. Integrative analysis reveals selective 9p24.1 amplification, increased PD-1 ligand expression, and further induction via JAK2 in nodular sclerosing Hodgkin lymphoma and primary mediastinal large B-cell lymphoma. Blood. 2010;116:3268-77.

72. Ansell SM, Lesokhin AM, Borrello I, Halwani A, Scott EC, Gutierrez M, Schuster SJ, Millenson MM, Cattry D, Freeman GJ, et al. PD-1 blockade with nivolumab in relapsed or refractory Hodgkin's lymphoma. N Engl J Med. 2015;372:311-9.

73. Westin JR, Chu F, Zhang M, Fayad LE, Kwak LW, Fowler N, Romaguera J, Hagemeister F, Fanale M, Samaniego F, et al. Safety and activity of PD1 blockade by pidilizumab in combination with rituximab in patients with relapsed follicular lymphoma: a single group, open-label, phase 2 trial. Lancet Oncol. 2014;15:69-77.

74. Lesokhin AM, Ansell SM, Armand P, Scott EC, Halwani A, Gutierrez M, Millenson MM, Cohen AD, Schuster SJ, Lebovic D, et al. Nivolumab in patients with relapsed or refractory hematologic malignancy: preliminary results of a phase lb study. J Clin Oncol. 2016;34:2698-704.

75. Bonanno G, Mariotti A, Procoli A, Folgiero V, Natale D, De Rosa L, Majolino I, Novarese L, Rocci A, Gambella M, et al. Indoleamine 2,3-dioxygenase 1 (IDO1) activity correlates with immune system abnormalities in multiple myeloma. J Transl Med. 2012;10:247.

76. Replogle JM, De Jager PL. Epigenomics in translational research. Transl Res. 2015;165:7-11.
77. Hamm CA, Costa FF. Epigenomes as therapeutic targets. Pharmacol Ther. 2015;151:72-86.

78. Arribas AJ, Rinaldi A, Mensah AA, Kwee I, Cascione L, Robles EF, MartinezCliment JA, Oscier D, Arcaini L, Baldini L, et al. DNA methylation profiling identifies two splenic marginal zone lymphoma subgroups with different clinical and genetic features. Blood. 2015;125:1922-31.

79. Hamm CA, Costa FF. The impact of epigenomics on future drug design and new therapies. Drug Discov Today. 2011;16:626-35.

80. Robertson KD. DNA methylation and human disease. Nat Rev Genet. 2005;6:597-610.

81. Krueger F, Kreck B, Franke A, Andrews SR. DNA methylome analysis using short bisulfite sequencing data. Nat Methods. 2012;9:145-51.

82. Roadmap Epigenomics Project. http://www.roadmapepigenomics.org/. Accessed 15 Aug 2016.

83. Blum KA, Liu Z, Lucas DM, Chen P, Xie Z, Baiocchi R, Benson DM, Devine $S M$, Jones J, Andritsos L, et al. Phase I trial of low dose decitabine targeting DNA hypermethylation in patients with chronic lymphocytic leukaemia and non-Hodgkin lymphoma: dose-limiting myelosuppression without evidence of DNA hypomethylation. Br J Haematol. 2010;150:189-95.

84. Clozel T, Yang S, Elstrom RL, Tam W, Martin P, Kormaksson M, Banerjee S, Vasanthakumar A, Culjkovic B, Scott DW, et al. Mechanism-based epigenetic chemosensitization therapy of diffuse large B-cell lymphoma. Cancer Discov. 2013;3:1002-19.

85. Stathis A, Hotte SJ, Chen EX, Hirte HW, Oza AM, Moretto P, Webster S, Laughlin A, Stayner LA, McGill S, et al. Phase I study of decitabine in combination with vorinostat in patients with advanced solid tumors and non-Hodgkin's lymphomas. Clin Cancer Res. 2011;17:1582-90.

86. Pon JR, Marra MA. Clinical impact of molecular features in diffuse large B-cell lymphoma and follicular lymphoma. Blood. 2016;127:181-6.

87. Lenhard B, Wasserman WW. TFBS: computational framework for transcription factor binding site analysis. Bioinformatics. 2002;18:1135-6.

\section{Submit your next manuscript to BioMed Central and we will help you at every step:}

- We accept pre-submission inquiries

- Our selector tool helps you to find the most relevant journal

- We provide round the clock customer support

- Convenient online submission

- Thorough peer review

- Inclusion in PubMed and all major indexing services

- Maximum visibility for your research

Submit your manuscript at www.biomedcentral.com/submit
() Biomed Central 